\title{
Caracterización clínica y estado actual de los pacientes con Síndrome de Asperger de 0 a 16 años, atendidos en la Fundación Liga Central Contra la Epilepsia y el Hospital de la Misericordia de Bogotá, Colombia
}

\author{
Ricardo Andrés Naranjo Flórez MD., Esp. \\ Médico Neuropediatra, Universidad Nacional de Colombia.
}

Correspondencia: andresnaranjoflorez@gmail.com

Recibido: 14/03/2014 Aceptado: 17/05/2014

\begin{abstract}
Resumen
El objetivo del presente estudio fue determinar los hallazgos clínicos, paraclínicos, epidemiológicos, y de pruebas de tamizaje y de diagnóstico en el síndrome de Asperger, en los casos que se detectaron en la Fundación Liga Central Contra La Epilepsia y la Fundación Hospital de la Misericordia entre los años 2004 y 2007. Hasta la fecha existen pocos artículos de series clínicas sobre este síndrome en la literatura médica, se ha informado que es un trastorno sub-diagnosticado en Colombia. Este estudio describe el síndrome con detalle, mostrando sus características en nuestra población, con el fin de ofrecer una mejor información para diagnóstico, tratamiento, seguimiento y prevención.
\end{abstract}

Palabras clave: autismo, comorbilidad, neurodesarrollo, Síndrome de Asperger.

\section{Clinical characterization and current status of the patients with Asperger's Syndrome from $\mathbf{0}$ to 16 years, served by the Foundation Central League Against Epilepsy and the Hospital of Mercy, Bogotá, Colombia}

\begin{abstract}
The objective of this study was to determine the clinical, paraclinical, epidemiological, and screening tests and diagnostic findings in Asperger syndrome, in the cases that were detected in the Central Foundation League Against Epilepsy and the Foundation of the Hospital of Misericordi between the years 2004 and 2007. To date, there are few articles of clinical series on this syndrome in the medical literature, it has been reported that it is a sub-diagnosed disorder in Colombia. This study describes the syndrome with detail, showing all their characteristics in our population, in order to provide better information for diagnosis, treatment, follow-up and prevention.
\end{abstract}

Key Words: Asperger Syndrome, Autism, comorbidity, neurodevelopment. 


\section{Introducción}

El síndrome de Asperger es un trastorno generalizado del desarrollo, descrito por Hans Asperger en 1944 , que se caracteriza por marcada alteración social, dificultades en comunicación, déficit en capacidad de juego y un rango de comportamiento e intereses repetitivos, sin un retardo significativo en el lenguaje, ni cognitivo. Sus características clínicas se establecieron hasta 1981 por la doctora Wing. Solo fue incluido como diagnóstico en el Manual Diagnóstico y Estadistico de los Trastornos Mentales (DSM-IV) en 1994. La prevalencia exacta no se ha determinado, es particularmente difícil de estimar y solo se encuentran aproximaciones, pero es más frecuente en hombres. Estas tasas de prevalencia para el Asperger van de 0.3 a 48 por 10.000 , con una media de 36 por 10000 nińos, nacidos vivos, por año (1-54).

Se han realizado numerosos intentos para establecer criterios diagnósticos claros, que se reflejan en el consenso del DSMIV. Como es una enfermedad crónica del comportamiento, no existe un patrón único de comorbilidad depende de las interacciones entre ambiente, herencia y paciente (1-48). Se desconocen los factores de riesgo, como antecedentes familiares, prenatales, perinatales o personales que puedan modificar el patrón genético. Aún está en estudio el patrón de herencia, pero hay datos que sugieren una fuerte relación(50-52).

El síndrome de Asperger se ha diferenciado muy recientemente del autismo de alto funcionamiento y existe poca información sobre el pronóstico de estos niños. En la medida en que los diagnósticos pudieran presumirse con el mínimo de exámenes y de tiempo, podría adelantarse una terapéutica encaminada a disminuir el impacto de la enfermedad (52-53). De esta forma, es importante establecer un diagnóstico temprano del Síndrome de Asperger para poder instaurar un tratamiento adecuado y mejorar el pronóstico, al evitar la comorbilidad. El cuadro clínico que se presenta en este síndrome está influenciado por múltiples factores. Es una enfermedad multifactorial, que no tiene una causa única, su etiología por consiguiente es compleja (1-48).

Debido a que hasta la fecha existen pocos artículos de series clínicas sobre este síndrome en la literatura médica, posiblemente es un trastorno sub diagnosticado en Colombia. Este estudio pretende describir el síndrome con más detalle, mostrando sus características en nuestra población, con el fin de ofrecer una mejor información para diagnóstico, tratamiento, seguimiento y prevención (49).

\section{Materiales y métodos}

Se diseñó un estudio descriptivo de serie de casos. Se tomó una muestra de 19 pacientes que previamente habían sido diagnosticados como Síndrome de Asperger, remitidos a la fundación Liga contra la Epilepsia y Fundación Hospital de la Misericordia, por neuropsicólogos, psiquiatras infantiles y neuropediatras. Se realizó una historia clínica completa a los pacientes, observación directa, entrevista a los padres con 3 sesiones de 90 minutos cada una, realizándose confirmación de caso, con uso de criterios diagnósticos de DSM-IV, CIE-10 y de Gillberg, con posterior aplicación de escala de tamizaje con el cuestionario de muestreo para espectro autista de alto funcionamiento (ASSQ).

Posteriormente, se realizó prueba diagnóstica para Síndrome de Asperger utilizando escala australiana para diagnóstico de Síndrome de Asperger (ASAS) y evaluación física de los pacientes, aplicación de instrumento de recolección de datos y evaluación de estudios paraclínicos previos. Se creó una base de datos en Excel, para posterior análisis estadístico con el programa SPSS, versión 10.0. Se hizo análisis de estadísticos descriptivos de frecuencias de tipo uni y multivariado. 


\section{Resultados}

El Síndrome de Asperger predomina en el sexo masculino, es detectado por los padres hacia los 3 años por dificultad en los procesos de socialización y adquisición tardía del lenguaje, se diagnostica cerca de los 9 años, Figuras 1 y 2.

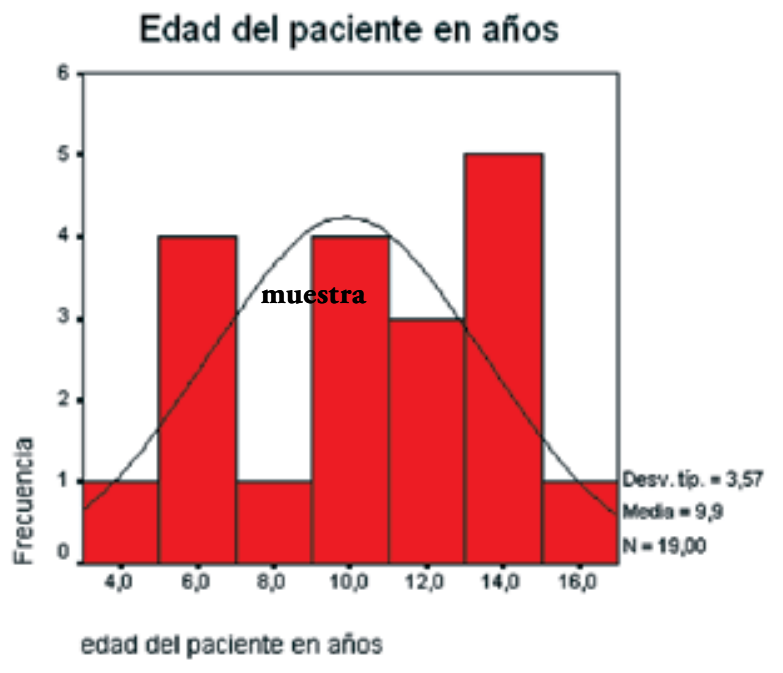

Figura 1. Se muestra la distribución de edad en los pacientes con Síndrome de Asperger participantes en el estudio.

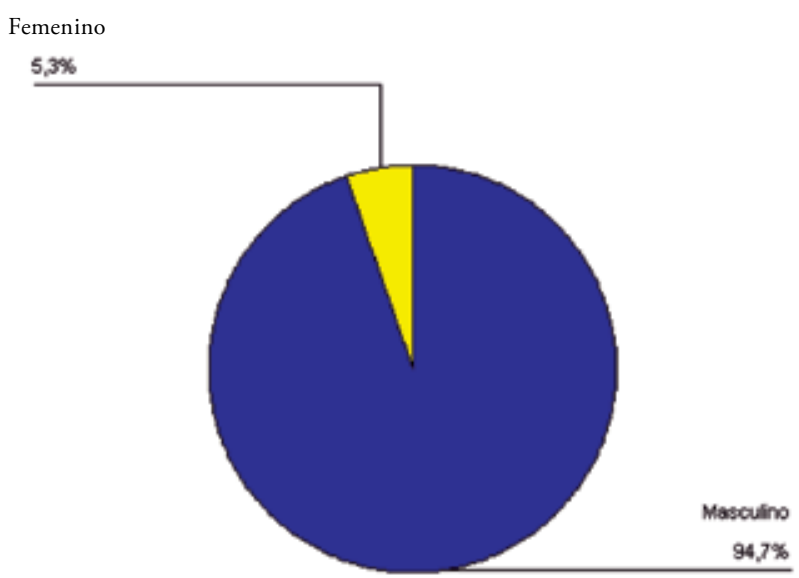

Figura 2. Distribución por género en pacientes con Síndrome de Asperger.

En cuanto al grado de escolaridad observado en los pacientes, se documentó que el $10.5 \%(\mathrm{n}=2)$ se encontraban en preescolar, el 63.2\% $(n=12)$ en primaria y el $26.3 \%(n=5)$ restante se encontraban cursando bachillerato; el mayor porcentaje se encontró en cuarto de primaria $21.1 \%$ $(n=4)$, Figura 3.

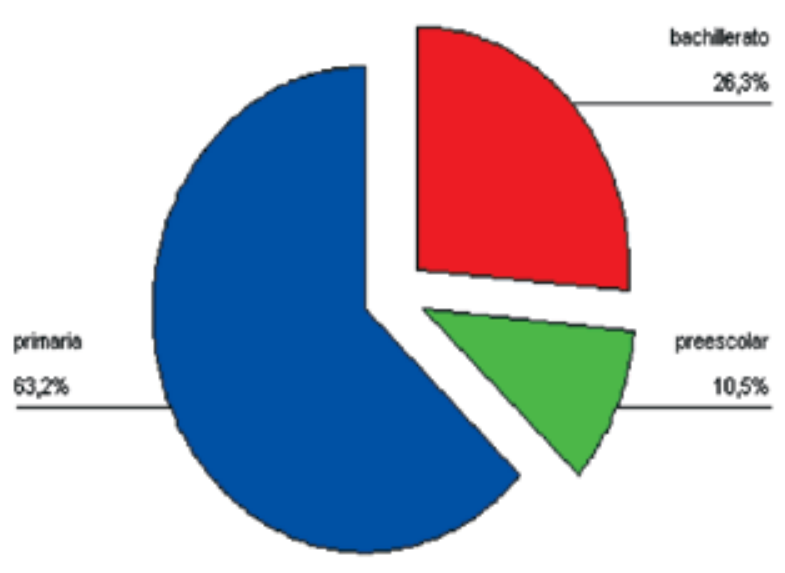

Figura 3. Grado de escolaridad de los pacientes con Síndrome de Asperger.

Los síntomas detectados fueron auto y heteroagresividad, impulsividad, abuso por sus pares, trastorno de tic, trastornos emocionales, alteración en control de esfínteres y trastorno de sueño. La edad de inicio de los síntomas tuvo una media de 3.14 años, una mediana de 2.5 años, con una desviación típica de 3.05 años, con un rango de aparición entre los 3 años y los 15 años. Los síntomas se notaron antes del año de edad en el $5.3 \%(n=1)$ de los casos, entre el año y los 2 años de vida se detectaron en un $15.8 \%(\mathrm{n}=3)$, siendo más frecuente a los 3 ańos $(31.6 \%, 6$ pacientes). De los 2-4 años se detectaron en el 63.2 $\%(n=12)$, Figura 4.

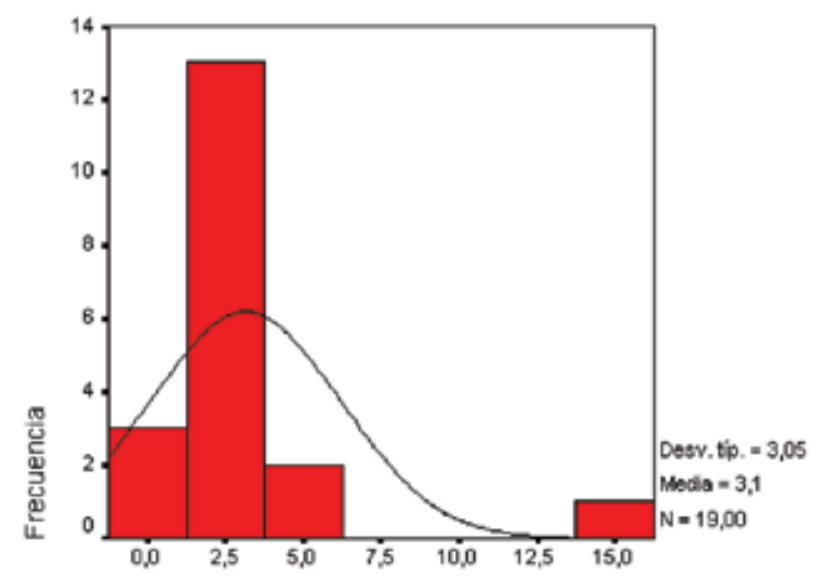

Figura 4. Edad de inicio de síntomas en los pacientes con Síndrome de Asperger. 
El motivo principal de consulta fue la dificultad en socialización evidenciada en el $47.4 \%(n=9)$ seguida de la adquisición tardía del lenguaje en el $36.8 \%$ ( $\mathrm{n}=7$ ). Otros motivos de consulta fueron movimientos estereotipados, ansiedad y convulsiones en el restante $15.9 \%(n=3)$, Figura 5 .

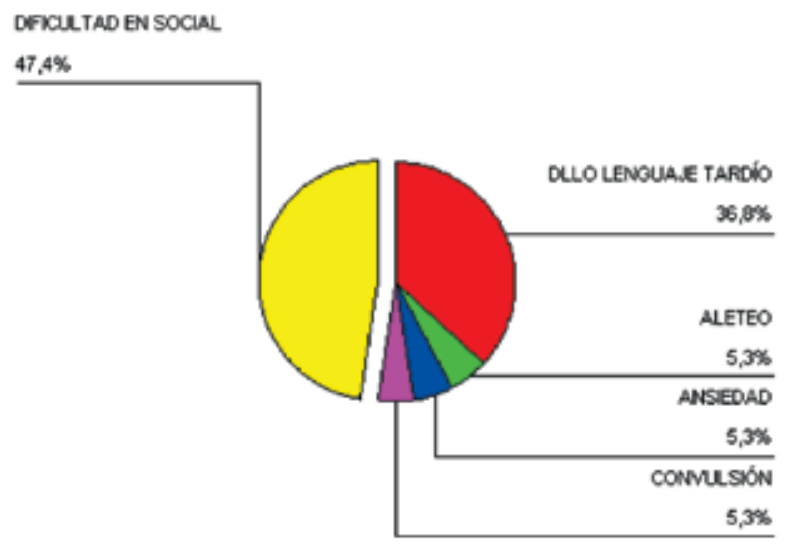

Figura 5. Motivo de consulta de los pacientes con Síndrome de Asperger.

La mayoría de los pacientes fueron fruto de primera y única gestación con embarazo controlado, de curso asintomático; parto instrumentado o por cesárea indicada por causa materna en la mayoría de los casos, la adaptación neonatal fue inmediata y espontánea. Figura 6. Todos los pacientes mostraron retardo en neurodesarrollo y una tercera parte presentó procesos alérgicos.

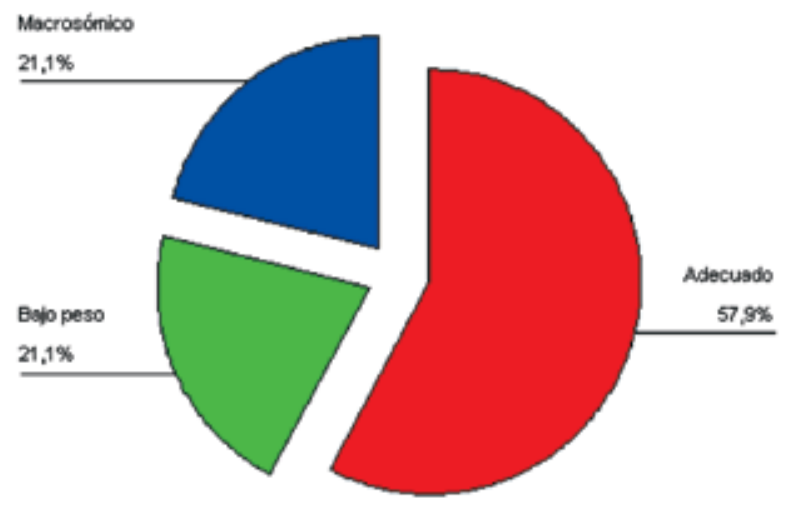

Figura 6. Peso al nacer de los pacientes on Síndrome de Asperger.
Se documentó epilepsia y EEG anormal en el $10 \%$. Las neuroimágenes no mostraron anomalías específicas, solo se documentó asimetría ventricular como hallazgo incidental. Se demostró hipoacusia conductiva en el $15 \%$ de los pacientes. Así mismo, se encontró una traslocación robertsoniana $\mathrm{t}(13 ; 14)$ en un paciente.

El 63\% presentó macrocráneoen relación con lo reportado en la literatura actual (55-57). En cuanto a movimientos anormales se presenta una comorbilidad con trastorno de tics en el $79 \%$, comorbilidad psiquiátrica en el $66 \%$, dada por trastornos de ansiedad, principalmente el trastorno obsesivo-compulsivo.

Se utilizaron medicaciones en el 32\% de los pacientes y de estos un $66 \%$ presentó respuesta adecuada, Figura 7.

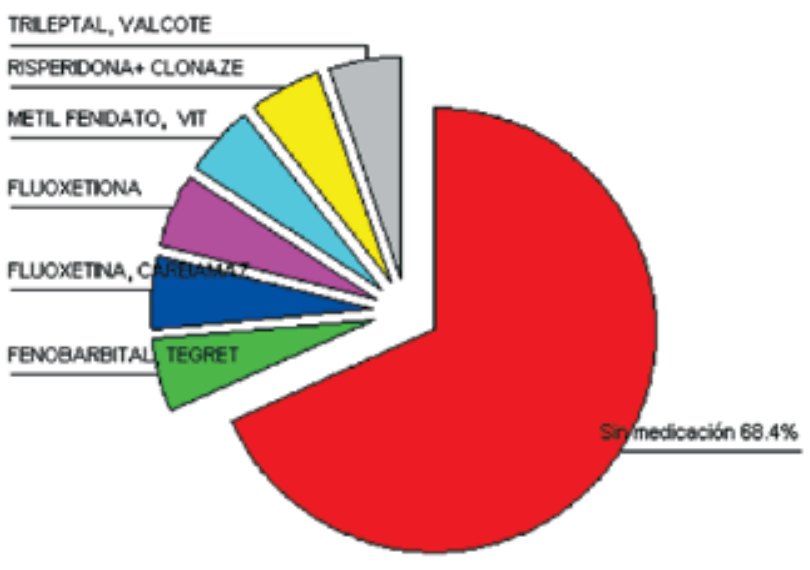

Figura 7. Medicaciones utilizadas en los pacientes con Síndrome de Asperger.

En cuanto a la media del puntaje total de La Escala ASSQ fue de 33,63, es decir positiva en todos los casos con un mínimo de 23 y un máximo de 41, con un punto de corte para positividad de 19 cuando la escala es diligenciada por los padres. La mediana y la moda fueron iguales y correspondieron a 34 . El intervalo de los datos fue de 30.98 a $36.28 \pm 5,50$, Figura 8 . 


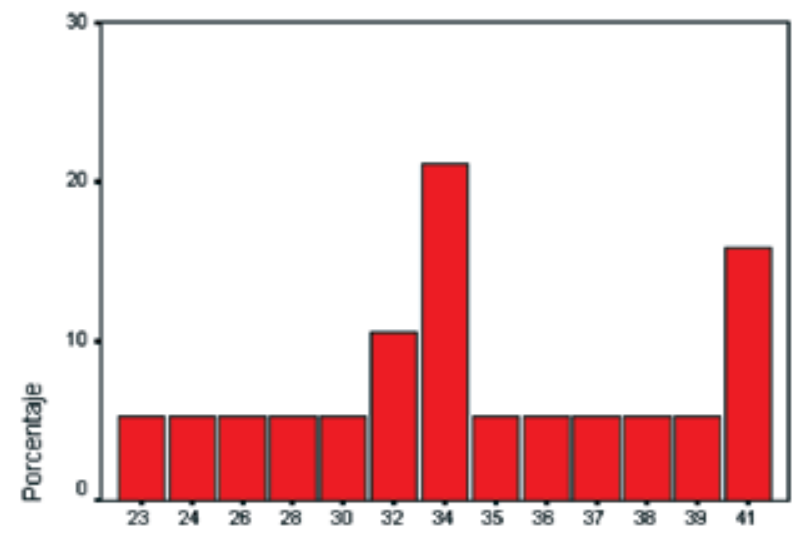

Figura 8. Distribución porcentual de los puntajes en la escala ASSQ.

De esta forma, el 57.9\% puntuó entre 23 y 34, el $21.5 \%$ entre 35 y 40 y $15.8 \%$ más de 40 . La mayor frecuencia de los valores se obtuvo entre 32 y 34 $(31.6 \%, n=6)$. El histograma, graficado con curva normal, muestra asimetría negativa de(-0.479), que indica que los valores más extremos se encuentran por debajo de la media y una curtosis con tendencia negativa de (-0.505), pero próxima a cero, acercándose a la curva de normalidad y mostrando que la mayoría de los casos se encuentra cerca de la media, Figura 9.

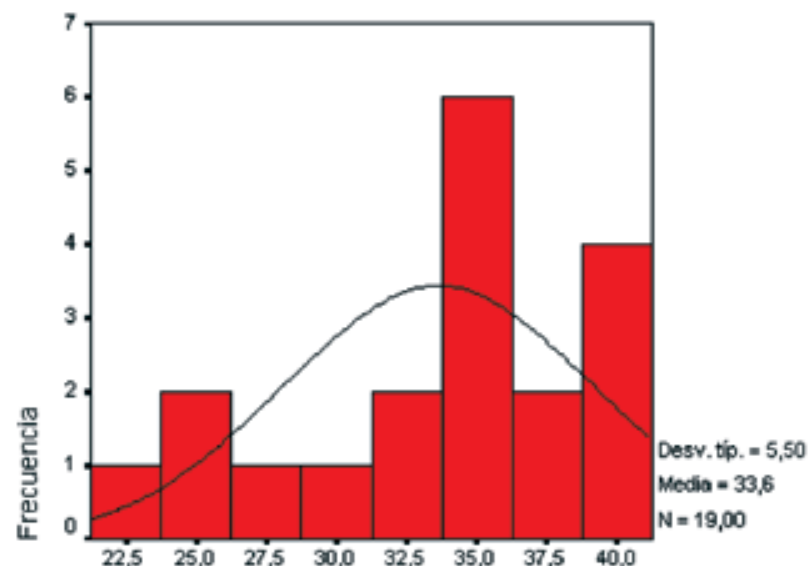

Figura 9. Histograma con línea normal que muestra la media de la distribución.

En cuanto a calificación sobre cada uno de los ítems evaluados en la escala, se encontró que 20 de 27 preguntas de la encuesta resultaron respuestas positivas. La respuesta positiva más frecuente fue sobre acumulación de datos sobre tópicos restringidos, mientras que la menos sensible fue sobre expresión facial inusual.
La media del puntaje total de La Escala ASAS fue de 97.21, es decir positiva en todos los casos, con un mínimo de 70 y un máximo de 110 puntos, con un punto de corte para positividad de 48. El valor de la media de la distribución fue de 97.21, mientras el valor de la moda fue 107, en un rango entre 91.34 y 103.08 con una desviación típica de 12.17 puntos. Figura 10.

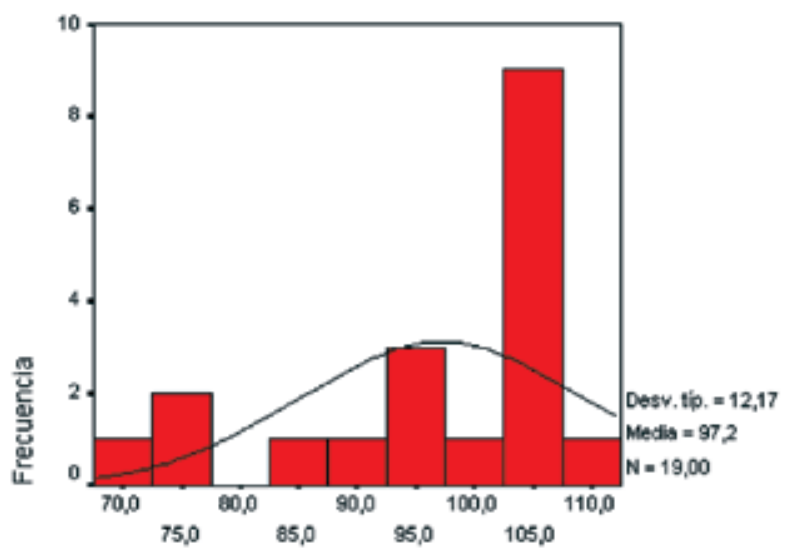

Figura 10. Distribución de puntaje en Escala ASAS.

En cuanto a calificación sobre cada uno de los ítems evaluados en la escala se encontró que las 24 preguntas evaluadas fueron positivas. La pregunta que obtuvo el puntaje más alto en severidad fue sobre búsqueda de información compulsiva sobre tópico restringido, con una media de 5.05; mientras que la pregunta con el puntaje de severidad más bajo fue sobre expresión anormal de la emoción con 2.68 .

Los puntajes más altos en las escalas se encuentran en pacientes con antecedentes de embarazo con patología obstétrica, anormalidades en período neonatal, alteración en neurodesarrollo, historia familiar sugestiva de conductas similares y con coeficientes intelectuales en rango normal a bajo; estos pacientes eran quienes tenían mayor comorbilidad y necesitaban medicación. 


\section{Discusión}

Este es el primer trabajo colombiano que describe las características de los pacientes con Síndrome de Asperger, y define las posibles características propias de los pacientes evaluados por los grupos de Neuropediatría y Psiquiatría Infantil de la Universidad Nacional de Colombia.

La edad promedio de los pacientes participantes en el estudio fue de 9.78 años. Se evaluaron pacientes desde los 3 hasta los 15 años, aunque la mayoría del grupo contaba con más de seis ańos y eran hombres. En cuanto a la escolaridad, es importante resaltar que la mayoría de los pacientes se encontraban en el grado adecuado para su edad pese a las dificultades que afrontan en el proceso educativo, como se discutirá más adelante, Figura 3. (51,52).

Los síntomas fueron detectados por los padres desde los 3 años y fueron diagnosticados por los profesionales de la salud hacia los 9 años, en concordancia con lo reportado por Mats y Gillberg en 2004 (22). Preocupa que pese a una temprana aparición de síntomas, que se convierte desde este momento en preocupación para los padres, el diagnóstico y la intervención clínica se realice tardíamente (22). Pero sin duda, se evidenció que se trata de una enfermedad compleja, que requiere habilidades y destrezas específicas de los médicos, Figura 4.

El síntoma más llamativo para los padres fue la dificultad para socializar. Esta dificultad se asocia a una adquisición tardía del lenguaje comparable con los datos suministrados por Howlin en 1999 (15). Desafortunadamente este síntoma, aparición tardía del lenguaje, en muchas oportunidades es subvalorado por el sistema de salud e incluso por el grupo familiar, Figura 5.

La mayoría de los pacientes fueron fruto de primera gestación única, controlada por un medico y de curso asintomático. En la minoría de los casos se documentaron patologías obstétricas relacionadas cerca del 30\%, similar a lo encontrado por Mats y Gillberg en 2004 (22). Este hallazgo está en concordancia con la escasa relación con factores de riesgo para lesión cerebral.

La mayor parte de los partos fueron por cesárea con indicación por distocia materna realizándose de forma programada, con una adaptación neonatal inmediata espontánea y en una cuarta parte de los casos, se asoció con pobre succión en el período neonatal, sin evidencia de encefalopatía posterior, lo que muestra que esta diferencia en el proceso del parto no fue factor de riesgo para suponer daño cerebral. Generalmente la cesárea se asocia a lesión cerebral posterior, en relación con lo reportado con Eaton, en 2001. Este autor puso en evidencia la relación de cesárea con problemas de socialización y aprendizaje. Pero igualmente es cierto que con los cambios que introdujo la Ley 100 en la atención sanitaria de los pacientes, una muy alta proporción de estos nacen por cesárea (28).

En cuanto al neurodesarrollo, aparece desviado en todas las áreas. Este aspecto es el motivo de los estudios y de la intervención en la mayoría de los pacientes. Aunque los criterios del DSM IV no incluyen el retardo del desarrollo del lenguaje, para esta serie el $100 \%$ de los pacientes lo presentaron, en concordancia con los criterios modificados de Gillberg. Tampoco se registraron antecedentes patológicos relevantes que explicaran las alteraciones del desarrollo.

No se registraron antecedentes familiares de autismo. Sin embargo resulta llamativo que muchos de los padres de estos niños refirieran timidez y síntomas obsesivos. Ellos describen síntomas similares en su infancia o incluso en su vida adulta, como aislamiento social, referido como "timidez", conductas repetitivas interpretado como "muy ordenados" lo que sugiere casos de sintomatología de interferencia escasa o que, nuevamente, por las dificultades diagnósticas, nunca se realizó el diagnóstico correcto, Figura 11. 


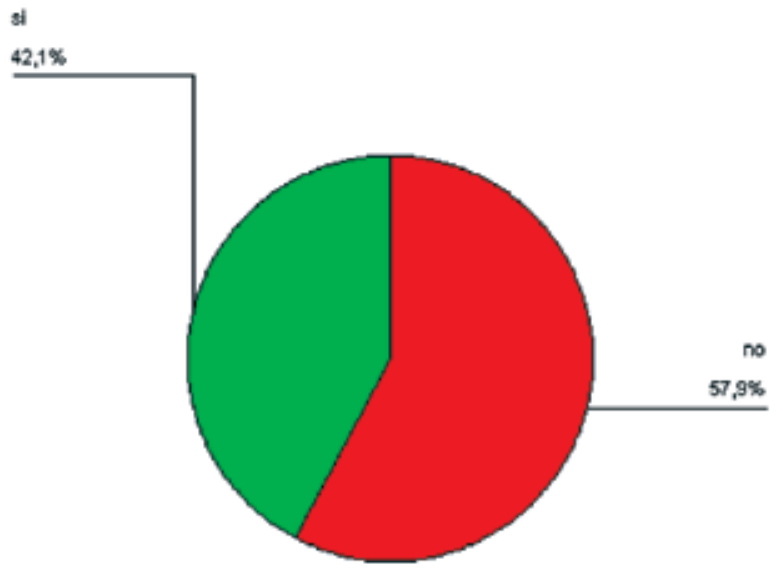

Figura 11. Familiares con conductas similares.

Los pacientes refirieron miedo o angustia inusual a los ruidos fuertes en el $47.4 \%$ ( $n=9)$, seguido de los sonidos ordinarios, como electrodomésticos o automotores en el 21.1\% ( $\mathrm{n}=4)$, además de los lugares ruidosos o atestados en el $10.5 \%(n=5)$, seguidos de caricias suaves en el 5.3\% $(\mathrm{n}=1)$, revelando inmadurez táctil, Figura 12.

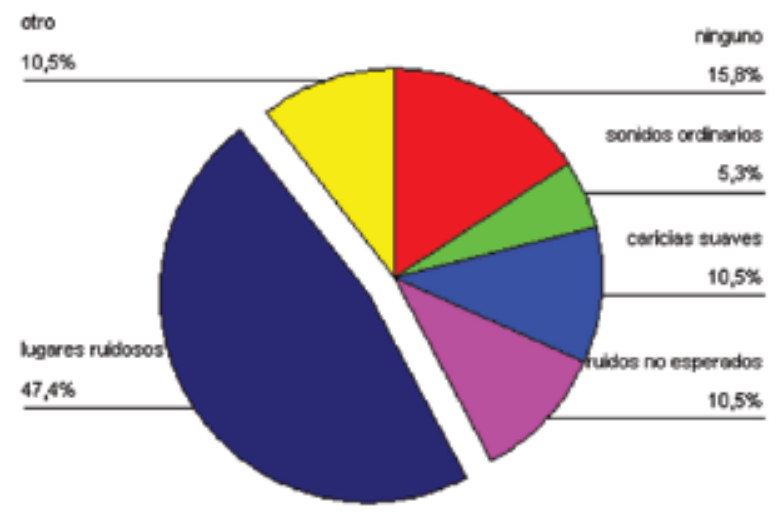

Figura 12. Miedos inusuales en pacientes con Síndrome de Asperger.

Las intervenciones farmacológicas mostraron buena respuesta para el tratamiento de síntomas específicos, como lo eran: depresión, inquietud y agresividad, en dos terceras partes de los pacientes. Las terapias de estimulación fueron de utilidad en un tercio de esta población. Aquí es importante tener en cuenta el objetivo con cada una de las medidas terapéuticas y las expectativas de mejoría de acuerdo al caso específico, Figura 7.

En estos pacientes el objetivo principal del tratamiento es facilitar la socialización, buscando mejores estrategias de interacción y comunicación, facilitando la flexibilidad en su comportamiento. Si este no fue el objetivo de padres, terapeutas y médicos, es poco probable que estas intervenciones surtieran algún efecto benéfico.

La comorbilidad psiquiátrica se documentó en dos terceras partes de los pacientes, en concordancia con valores previos cercanos al $60 \%$, siendo más frecuente los síntomas de ansiedad, entre ellos el trastorno obsesivo-compulsivo, similar a lo reportado por varios autores $(16,21,33,38,41-45)$. Los temas que se convierten en obsesivos son internet, mapas, carros, aviones, etc. Lucen ansiosos ante pequeños cambios o cuando se producen modificaciones en la rutinas cotidianas.

La asociación con epilepsia fue 8 veces mayor que en la población general y el EEG anormal en el $10 \%$ de los casos. Este dato está en contraposición con el 30\% documentado para autismo general. Si bien no siempre este hallazgo explica la alteración en el desarrollo, en los casos donde se evidencie involución o deterioro en el desarrollo y / o lenguaje, debe considerarse como diagnóstico diferencial los síndromes epilépticos del espectro de la epilepsia de punta onda continua en sueño profundo o ESES, lo que amerita intervención con anticonvulsivos.

El coeficiente intelectual de los pacientes fue normal, puntuó entre normal bajo y normal alto. La gran preocupación está en que las pruebas estandarizadas para niños evalúan ante todo el desarrollo del lenguaje, siendo este aspecto el más afectado en ellos. Por esta razón se empiezan a utilizar pruebas especiales con estrategias que no comprometan los aspectos de lenguaje, Figura 13. 


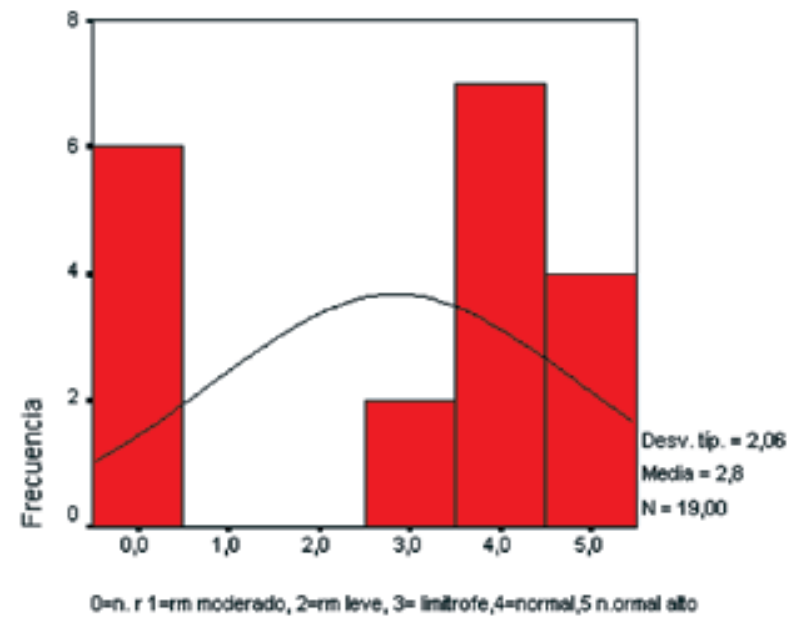

Figura 13. Distribución de los puntajes de coeficiente intelectual.

El 63\% presentó macrocráneo, mayor a lo reportado por Gillberg del $30 \%$ en 2002 . Aunque no existe un dato exacto acerca de la relación entre macrocráneo y alteraciones del desarrollo, esta relación se ha documentado frecuentemente $(56$, 57). Aunque este aumento del perímetro cefálico no indica lesión estructural específica, puede ser un síntoma que amerite vigilancia específica de un paciente en cuanto a su desempeño social o del lenguaje.

Las neuroimágenes no mostraron anomalías específicas, solo se documentó asimetría ventricular, como hallazgo incidental, similar a lo evidenciado por Aviva en 1999 y otros autores (2, 4, 16-23). Si bien no existen alteraciones anatómicas específicas que sean evidenciables y confirmen el diagnóstico, existe la posibilidad de avanzar en estudios funcionales con protocolos específicos encaminados a detectar anomalías semejantes en el Síndrome de Asperger.

Se demostró hipoacusia conductiva en el 15\% de los pacientes, comparado con lo registrado de autismo general del $10-20 \%$, en el mismo rango que observó Blacher en el 2003 (21,46). Esta baja frecuencia indica que las dificultades del lenguaje en la mayoría de los pacientes correspondían a alteraciones propias en el desarrollo de esta habilidad y no por un déficit sensorial. Entre los diagnósticos diferenciales se encuentran las disfasias, siendo el trastorno del lenguaje de tipo semántico pragmático, por sus características, una de las más grandes dificultades en el diagnóstico.

Se documentó un caso corroborado de translocación robertsoniana, t $(13 ; 14)$, como hallazgo genotípico en un paciente de la muestra, en concordancia con otras translocaciones también robertsonianas descritas por otros autores (34).

Se determinó trastorno de tic en el 79\%, valor cercano al $80 \%$, reportado en la literatura mundial $(16,21,33,38,41-45)$. No es claro si este hallazgo corresponde a una característica más del síndrome o si es parte de la comorbilidad de estos pacientes, este es uno de los síntomas que requieren tratamiento específico para facilitar la integración de estos pacientes a su entorno. Para efectos prácticos se puede asumir que los tics en estos pacientes son síntomas ansiosos. Otros de los síntomas encontrados fueron alteraciones en la percepción tanto auditiva o táctil, orientando a considerar que estos pacientes muestran inmadurez en entradas sensoriales. Sin embargo, no se registran lesiones en áreas de integración sensorial y no muestran mejoría con terapias orientadas a este aspecto, lo que genera confusión en cuanto al posible mecanismo neurofisiológico relacionado y como poder enfrentar esta dificultad.

Es muy llamativo encontrar que estos pacientes con buen desempeño escolar, tenían en el $50 \%$ de ellos trastornos del aprendizaje (lectura y escritura). Sólo un tercio de los pacientes hace amistad con sus pares fácilmente, un tercio es sometido a burlas y apodos y un tercio es invitado a fiestas infantiles. Esta distribución es similar a la reportada por Foster en 2004 (16) La combinación de dificultades en la socialización y expresión emocional limitan su posibilidad de desempeñarse adecuadamente en el ambiente escolar, llevando al fracaso en esta área, Figura 14. 


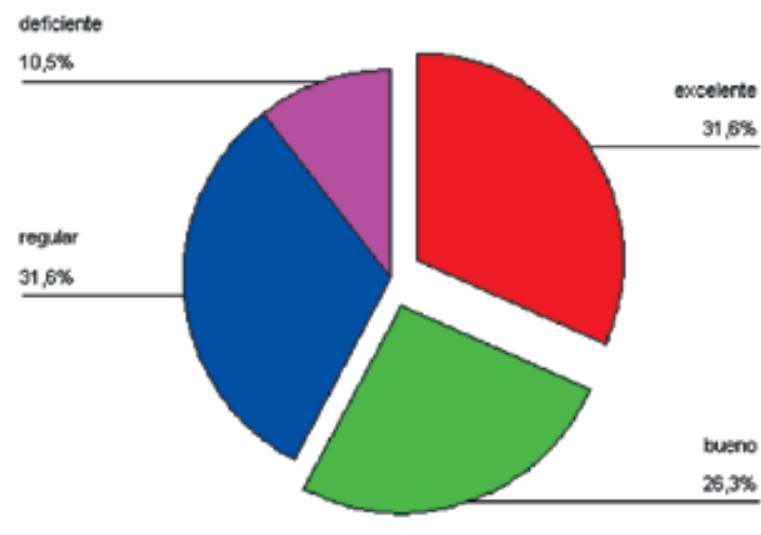

Figura 14. Rendimiento escolar en pacientes Asperger estudiados.

En cuanto a la evaluación realizada por las escalas, se encontró que todos los pacientes presentaron puntajes positivos. Preocupa la tendencia a puntajes altos, sugiriendo que son más fáciles de diagnosticar aquellos pacientes con síntomas más marcados y que aquellos cuyos síntomas son sutiles pueden estar pasando inadvertidos, en relacion con lo visto en las evaluaciones llevadas a cabo en otras latitudes (54-57).

El síntoma que fue más importante en ambas escalas fue el de los temas de interés restringido, siendo esta una de las características más llamativas de estos pacientes y que debe explorarse siempre que se considere este diagnóstico en un paciente, Figura 12.

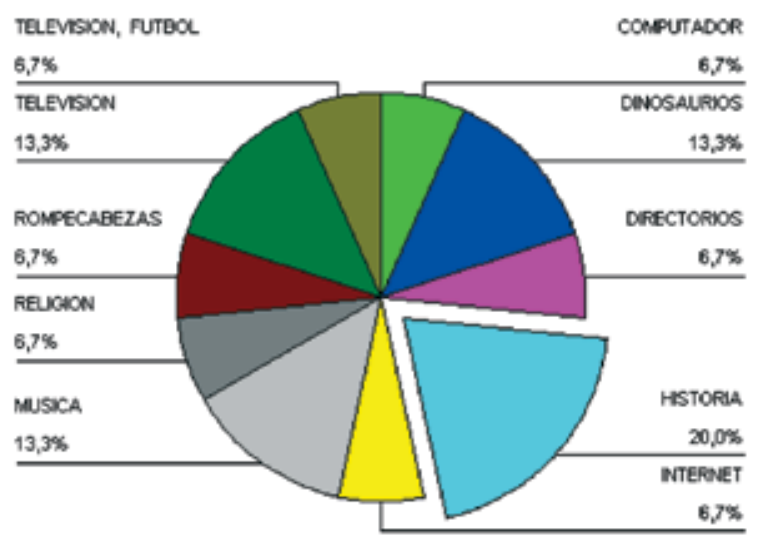

Figura 15. Tópicos de interés restringido en pacientes con Síndrome de Asperger.
Los puntajes más altos en la calificación de estas escalas se relacionaron con los factores de riesgo pre, peri o postnatales, en quienes presentaban patologías y mayor comorbilidad neurológica y psiquiátrica, con coeficientes intelectuales en rango bajo y más dificultades en su desempeño social y escolar, al igual que en quienes mostraron mala respuesta a las diferentes opciones terapéuticas. Esto indica que, si bien no hay evidencia que los riesgos de lesión cerebral no determinan la presencia del Síndrome, si puede ser un factor de complicación de los síntomas y de mayor dificultad para el tratamiento de estos niños.

Aunque es importante determinar el estrato socioeconómico y la escolaridad de lo padres, no en todos los pacientes pudo determinarse por lo que no se realizó un análisis estadístico de este dato.

En Colombia el Síndrome de Asperger tiene mayor prevalencia en el género masculino y se diagnostica tardíamente. Esto puede deberse a la falta de entrenamiento de los médicos y por consulta tardía de los padres. Sin embargo los síntomas son detectados por los padres hacia los 3 años y se diagnostica cerca de los 9 años, cuando se encuentran cursando los primeros años de primaria. Su diagnóstico es clínico pero las escalas ayudan a su detección. Se subdiagnostica, por su alta comorbilidad tanto psiquiátrica como neurológica, asociado a desviaciones en el neurodesarrollo, que desvían las intervenciones y generan sesgo.

Lo que más llama la atención a los padres es la dificultad en socializar, asociada a una adquisición tardía del lenguaje, el cual se completa antes de los 36 meses, persistiendo alteraciones específicas en prosodia, semántica, pragmática y sintaxis. Todos los pacientes mostraron retardo en neurodesarrollo, principalmente de desarrollo social y lenguaje. El diagnóstico diferencial más frecuente es el trastorno de lenguaje semántico pragmático. Los padres tienen dificultades en reconocer síntomas por problemas semejantes en su núcleo familiar. 
Los factores de riesgo no son determinantes de la patología, pero si agravan su sintomatología clínica, mostrando mayores puntajes en las escalas, lo que amerita mayor intervención de un equipo interdisciplinario. Los paraclínicos muestran anormalidades en relación con la historia personal. No hay una clara descripción de los síntomas, pero existen algunos que pueden ser marcadores importantes para vigilar el desempeño de un paciente como la presencia de tic, las alteraciones en el lenguaje, la macrocránea y la historia de control tardía del control de esfínteres.

La mayoría de pacientes recibió apoyo terapéutico, pero solo se logró adecuada respuesta en una tercera parte de los pacientes. Debe aclarase en el futuro cuales deben ser las intervenciones terapéuticas específicas para mejorar los aspectos de socialización de estos pacientes.

Se usaron por primera vez, en nuestro medio, escalas de tamizaje y de diagnóstico para el Síndrome de Asperger, como camino inicial a la estandarización y validación de las mismas. El presente estudió, por ser descriptivo, no pretende dar asociaciones estadísticas, pero si permite observar que las escalas utilizadas fueron útiles con instrumento de tamizaje y seguimiento.

Este estudio permitió la conformación de la asociación de padres de familia de paciente con Asperger, que ya cuenta con constitución legal, estatutos, junta directiva y reconocimiento fiscal, cuyos objetivos son diseño de estrategias para optimizar manejos en colegio y casa, acceso oportuno a diagnóstico y educación familiar, promover ante instancias de ley normas que faciliten la vida en sociedad de estos niños afectados, financiar y conseguir recursos para desarrollar actividades de fortalecimiento en relaciones sociales de los pacientes, compartir experiencias de manejo y conformar un grupo sólido de apoyo mutuo.

\section{Referencias}

1. Wolff Sula. The history of autism. European children and adolescence psychiatry. 2004; 13:201-308.

2. Klin Ami, Asperger syndrome: an update. Revista Brasilera de psiquiatría. 2003; 25(2): 103-109.

3. Klin Ami. Asperger syndrome, Infant mental health Journal. 2001; 22 (6): 676.

4. Asperger syndrome coalition of the United States. Asperger syndrome, Harvard mental health letter. 2005.

5. Volkmar, F. Asperger's Disorder. American Journal of Psychiatry. 2000; 157: 2 .

6. Atwood, Tony, Asperger syndrome. Orphanet enciclopedia. 2003.

7. Charman, Tony, The prevalence of autism spectrum disorders, European child and adolescence psychiatry. 2011; 249-256.

8. Wing Lorna, The epidemiology of Autistic Spectrum disorders: is the prevalence rising?. Mental retardation and developmental disabilities research reviews. 2002; (8) 151-161.

9. American Psychiatric Association. (1994). Diagnostic and statistical manual of mental disorders (4th ed.). Washington, DC: Author.

10. American Psychiatric Association. (2000). Diagnostic and statistical manual of mental disorders (4th ed., text rev.). Washington, DC: Author.

11. World Health Organization. The ICD-10 classification of mental and behavioural disorders. Geneva: World Health Organization; 1992.

12. Attwood Tony, Síndrome de asperger una guía para los padres y los profesionales 1997 editor Jessica Kingsly, pág. 176.

13. Webb, E. Prevalence of autistic spectrum disorder in children attending mainstream schools in a welsh education authority, Developmental Medicine and Child neurology 2003; 15: 377-381.

14. Fombonne, E, What is the prevalence of Asperger Disorder?, Journal of autism and Developmental disorders. 2001; 31:3.

15. Howlin P, The diagnosis of autism and Asperger Syndrome; findings from a survey of 770 families, Developmental Medicine and child Neurology. 1999; 41:831-839.

16. Foster B, Asperger syndrome: to be or not to be? Current opinion in Paediatrics. 2003;15: 491-494.

17. Aviva F, Characterization and comparison of autistic subgroups: 10 years experience with autistic children, Developmental medicine and child Neurology. 1999; 41:21-25.

18. Klin Ami, Asperger syndrome; Guidelines for assessment and Diagnosis, 1995, Learning Disabilities Association of America.

19. Smith Myles B, Introduction to the special Issue on Asperger, Focus on Autism and other developmental disabilities. 2002;17(3):130-131.

20. Bax M, Diagnoses made too late, Developmental medicine and child neurology. 1999; 41: 795.

21. Blacher J, Asperger syndrome and high functioning autism, Current Opinion in Psychiatry 2003; 16: 535-542.

22. Mats $\mathrm{C}$ and Gillberg C. One hundred males with asperger syndrome, Developmental medicine and child neurology. 2004; 46:652-660.

23. Smith Myles B, Asperger syndrome: An overview of characteristics, Focus on Autism and other developmental disabilities. 2002; 17(3): 132-137. 
24. Szatmari P, two year outcome of preschool children with autism and asperger syndrome, American journal of Psychiatry. 2000; 157: 1980-1987.

25. Naidu A, Diagnosis of Asperger syndrome in a 66yearold male presenting with depression, International psycho geriatrics. 2006; 18(1):171-173.

26. Kadesjö, B, Brief report: Autism and Asperger syndrome in seven year old children: a total population study, Journal of autism and developmental disorders.1999; 29(4): 327-331.

27. Walker D. Specifying PDD-NOS; a comparison of PDD-NOS, asperger syndrome, and autism, Journal of American academy o child and adolescence psychiatry. 2004; 43(2):172-180.

28. Eaton William, obstetric complications and risk for severe psychopathology en childhood, Journal of autism and developmental disorders. 2001;31(3):279-285.

29. Oktem F, functional Magnetic Resonance Image in children with asperger syndrome, Journal of Child Neurology. 2001;16(4): 252-256.

30. Hower K, Voxel-based morphometry elucidates structural neuroanatomy of high-functioning autism and Asperger syndrome, Developmental medicine and child neurology. 2004. 46: 760-764.

31. Casanova M, Asperger syndrome and cortical neuropathology, Journal of Child Neurology. 2002. 17:142-144.

32. Justin B, Forensic aspects of Asperger syndrome, the journal of forensic psychiatry and psychology. 2004;15(1): 96-197.

33. Volkmar F, Klin A, Nosological and genetic aspects of Asperger Syndrome, Journal of autism and developmental disorders. 1998; 28(5):457-463.

34. Tentler D A candidate region for Asperger syndrome define by two $17 \mathrm{p}$ breakpoints, European journal of human genetics. 2002;11:189-195.

35. Ylisaukko-oja-T, genome-wide scan for loci of Asperger syndrome, molecular psychiatry. 2004; 9:161-168.

36. Campbell J, Diagnostis Assement of Asperger's Disorder: a reviw of five third-party rating scales, Journal of Austism and developmental disorders, 2005; 35(1): 25-35.

37. Ehlers S, Gillberg C, Wing L, A screening questionnaire for Asperger syndrome and otherhigh- functioning austism specrum disorders in school age children, Journal or autism and developmental disorder. 1999; 29(2): 129 -141.

38. Attwood Tony, El syndrome de Asperger, una guía para la familia 2002, Editorial Paidós Iberica, Barcelona, Espańa.

39. Maj-Britt P, Gillberg C, autistic features in a total population of 7-9 year old children assessed by the ASSQ (autism spectrum screenin questionnaire), journal of child Psychology and Psychiatry. 2006; 47(2):167-175.

40. Dickerson S, Calhoun S, influence of IQ and age in childhood austism: lack of support for DSM-IV asperger's disorder; Journal of developmental and physical disabilities. 2004; 16,3: 257-272.

41. Blacher J, et al, Asperger syndrome and high functioning autism: research concerns and emerging foci, Current opinion in Psychiatry. 2003;16: 535-542.
42. Ghaziuddin M, Asperger syndrome: associated psychiatric and medical conditions, Focus on autism and other developmental disabilities. 2002; 17(3): 138-144.

43. Schatz A, et al, Brief report: attention differences in asperger syndrome, Journal of autism and developmental disorders. 2002; 34: 333-336.

44. Rusell E, et al, Anxiety and social worries in children with asperger syndrome, Australian an New Zealand journal of psychiatry. 2005; 39: 633-638.

45. Ghaziuddin M, Ghaziuddin N. Comorbidity of Asperger syndrome; a preliminary report. 1998; 42(4): 279-283.

46. Gillberg C. Autism and asperger syndrome: coesistence with other clinical disorders, Acta psychiatica scandinavica. 2000; 102: 321-330.

47. Gillberg C, Head circumference in autism, asperger syndrome and ADHD; a comparative study, Developmental medicine and chil neurology. 2002; 11: 296-300.

48. Little L. Peer victimizacion of children with asperger spectrum disorders, Journal of the American academy of child and adolescent psychiatry. 2001; 40(9):995-996.

49. Tara A. Lavelle, Milton C. Weinstein, Joseph P. Newhouse, Kerim Munir, Karen A. Kuhlthau, and Lisa A. Prosser. Economic Burden of Childhood Autism Spectrum Disorders. Pediatrics 2014; 133: 520-529.

50. El-Fishawy P., State M. The Genetics of autismo: Key Issues, Recent Findings, and Clinical Implications. Psychiatr Clin N Am. 2010; (33): 83-105.

51. Sarabeth Broder-Fingert, Amy Shui, Christian D. Pulcini, Daniel Kurowski, and James M. Perrin. Racial and Ethnic Differences in Subspecialty Service Use by Children With Autism, Pediatrics. 2013; 132: 94-100.

52. Trastornos del espectro autista. Puesta al día (I): introducción, epidemiología y etiología. D. Martín Fernández-Mayoralas, et al. Acta Pediatr Esp. 2013; 71(8): 217-223.

53. Kiah Bertoglio, Robert L. Hendren, New Development in Autism. Psychiatric Clinics of North America 2009; (32): 1-14.

54. Plauche C., Myers J. Identification and Evaluation of Children With Autism Spectrum Disorders. Pediatrics. 2007; 120; 118.

55. Rich Stoner, Ph.D., Maggie L. Chow, Ph.D., Maureen P. Boyle, Ph.D., Susan M. Sunkin, Ph.D., Peter R. Mouton, Ph.D., Subhojit Roy, M.D., Ph.D., Anthony Wynshaw-Boris, M.D., Ph.D., Sophia A. Colamarino, Ph.D., Ed S. Lein, Ph.D., and Eric Courchesne, Ph.D. Patches of Disorganization in the Neocortex of Children with Autism. New England Journal. 2014; 370: 1209-1219.

56. Chawarska K, Campbell D, Chen L, Shic F, Klin A, Chang J. generalized overgrowth in boys with autism. Archives of General Psychiatry. Jama Phsychiatry. 2011; 68: 1021-31.

57. Hazlett HC, Poe MD, Gerig G, et al. Early brain overgrowth in autism associ- ated with an increase in cortical surface area before age 2 years. Archives of General Psychiatry. Jama. 2011; 68: $467-76$. 